\title{
IJTC2012-61219
}

\section{SELF-ORGANIZATION AND FRICTION DURING SLIDING}

\author{
Pradeep L. Menezes ${ }^{1,3}$, ishore $^{1}$, Satish V. Kailas ${ }^{2}$ and Michael R. Lovell ${ }^{3}$ \\ ${ }^{1}$ Department of Materials Engineering, Indian Institute of Science, Bangalore 560012 , India \\ ${ }^{2}$ Department of Mechanical Engineering, Indian Institute of Science, Bangalore 560 012, India \\ ${ }^{3}$ Department of Industrial Engineering, University of Wisconsin-Milwaukee, Milwaukee, WI 53201
}

\begin{abstract}
In self-organized sliding processes, the surfaces align to each other during sliding. This alignment leads to a more ordered contact state and significantly influences the frictional behavior. To understand the self-organization sliding processes, experiments were conducted on a pin-on-plate reciprocating sliding tester for various numbers of cycles. In the experiments, soft magnesium pins were slid against hard steel plates of various surface textures (undirectional, 8-ground, and random). Experimental results showed that the transfer layer formation on the steel plates increased with increasing number of cycles for all surfaces textures under both dry and lubricated conditions. The friction also increased with the number of cycles under dry conditions for all of the textures studied. During lubricated conditions, the friction decreased for unidirectional and 8-ground surfaces and increased for random surfaces with the number of cycles. Furthermore, the friction and transfer layer formation depend on the surface textures under both dry and lubricated conditions during the first few sliding cycles. Later on, it is less dependent of surface textures. The variation in the coefficient of friction under both dry and lubrication conditions were attributed to the self-organization process that occurred during repeated sliding.
\end{abstract}

\section{INTRODUCTION}

When two surfaces slide against each other, various phenomena take place at the interface. For example, surface roughness changes at the interface, transfer layer (i.e., secondary structures) builds up on the surfaces, and damage/shearing occurs on the surfaces. These processes depend on various tribological testing conditions. Friction and wear are irreversible processes which normally lead to material deterioration. Under certain situations, friction can lead to selforganization or alignment of surface profiles at the frictional interface. In the self-organized state, the surfaces adjust to each other, leading to a more ordered state and lower energy dissipation rates. In such a state, the surface structure evolves until it reaches a certain stationary state referred to as the equilibrium roughness distribution. This self-organization process leads to steady state friction and wear performance [1, 2].

Prior efforts have been made to study the self-organization process for surfaces [1] during sliding. The self-organization process was analyzed based on the change in roughness at the interface $[1,2]$. The self-organization process during sliding as a function of surface texture is not well documented in the literature. The initial surface texture indeed plays an important role during the self-organization process. Moreover, the friction and transfer layer formation depends on initial surface texture $[3,4]$. The formation of the transfer layer at the interface also contributes to the self-organization process. Hence, in this investigation efforts have been made to study the selforganization process as a function of surface texture during sliding. The self-organization process is very critical during running-in or repeating sliding contacts. One such example is in metal forming process where the sheet metal can alter the initial surface structure of the die during multiple sliding contacts. This can alter the friction values by more than 200\% thus changing the initial surface structure of the die by the selforganization process.

\section{EXPERIMENTAL DETAILS}

To understand the self-organization process as a function of surface textures, three kinds of textures were produced on the steel plate surfaces. They are unidirectional, 8-ground and random. The unidirectional surfaces were prepared by grinding the plates against emery papers in a unidirectional fashion. The 8-ground surface was generated by moving the steel plate against emery papers along a path with the shape of an "8" for 500 cycles. For the unidirectional and 8-ground surfaces, the roughness was varied using different grits of empery papers. The random textures were generated on the steel plates by polishing the steel plate against the pad of a standard metallographic disc polishing machine. For the random surfaces, the roughness was varied using different abrasive powders.

Experiments were conducted using a pin-on-plate reciprocating sliding tester [4]. In the experiments, the pins 
were made of pure magnesium (99.98 wt. \%) and the counterpart plates were made of die steel. The pins were slid at a sliding velocity of $2 \mathrm{~mm} / \mathrm{s}$ against the prepared steel plate surfaces for a track length of $10 \mathrm{~mm}$ in the forward direction (first half cycle) and then backward direction (another half cycle) to the initial position for each sliding cycle. Experiments were conducted for a constant normal load of $35 \mathrm{~N}$ under both dry and lubricated conditions on each plate in an ambient environment. Under dry conditions five parallel wear tracks were obtained on the same steel plate. Each wear track was produced by a different number of reciprocating sliding cycles 1, 3, 5, 10 and 20. A single pin was used for all the five sliding cycles. For the lubricated tests, a drop of commercially available engine oil lubricant was applied to the surface of the same steel plate and the tests were performed with a new magnesium pin to obtain another five parallel wear tracks of different number of reciprocating sliding cycles (similar to the dry tests). Both the dry and lubricated tests were done on the same steel plate so that the results of the dry and lubricated experiments would exclude variations in surface roughness of the steel plates. The pins were slid both in perpendicular and parallel direction to the unidirectional grinding marks on the plate. Thus, four sets of topographic conditions were examined. After the tests, a scanning electron microscope (SEM) was used to reveal the morphology of the transfer layer formed on the steel plates. Roughness analysis of the steel plate surfaces was performed using an optical profilometer.

\section{RESULTS AND DISCUSSION}

Experiments were conducted on four kinds of surface textures, UPD (i.e., sliding is perpendicular to the unidirectional grinding marks), 8-ground, UPL (sliding is parallel to the unidirectional grinding marks), and random, for various numbers of cycles for a constant normal load under dry and lubricated conditions. Figures 1 (a) and (b) present the range in which the coefficient of friction values varied under the operating conditions examined. The range of surface roughness, $R_{\mathrm{a}}$, was varied between 0.01 and $0.6 \mu \mathrm{m}$ for the different textured surfaces. For a given texture, the average coefficient of friction did not substantially vary over this range of roughness. The error bars in the figure indicate the maximum and minimum values of the friction obtained for five experiments at a given condition. Each symbol on Fig. 1 refers to the average coefficient of friction of five roughnesses of the same texture. It can be observed that the coefficient of friction increases with number of cycles under dry conditions for all kinds of textures. Under lubricated conditions, the coefficient of friction decreases for UPD, 8-ground and UPL surfaces as a function of cycles. The randomly ground surfaces, in contrast, show an increase in friction with the number of cycles. Under dry condition, the coefficient of friction values are much higher when compared to that under lubricated conditions. It can also be observed that the coefficient of friction depends significantly on the die surface textures during the first few cycles. The friction was highest for the UPD case, followed by the 8ground, UPL case, and was the least for the randomly polished surfaces for the first few cycles. At higher number of cycles, the friction was less dependent of die surface texture. Another interesting point to note is that the friction values converge to a particular value under lubricated conditions. However, they do not converge to a particular value under dry conditions.

Scanning electron micrographs of the UPD and random surfaces when the magnesium pin slid at $1^{\text {st }}$ and $20^{\text {th }}$ cycles under dry conditions is shown in Fig. 2. A significant amount of magnesium was transferred to the steel plates under dry conditions. At lower magnification, it was found that the amount of transfer layer formed on the steel plate increased with increasing number of cycles. Under lubricated conditions (Fig. 3), although the transfer layer formation increases with increasing number of cycles, the amount is less when compared to that under dry conditions. SEM micrographs of the magnesium pins slid on various surface textures under dry conditions showed surface shearing on the pin. Under lubricated conditions, the intensity of surface shearing was significantly reduced.
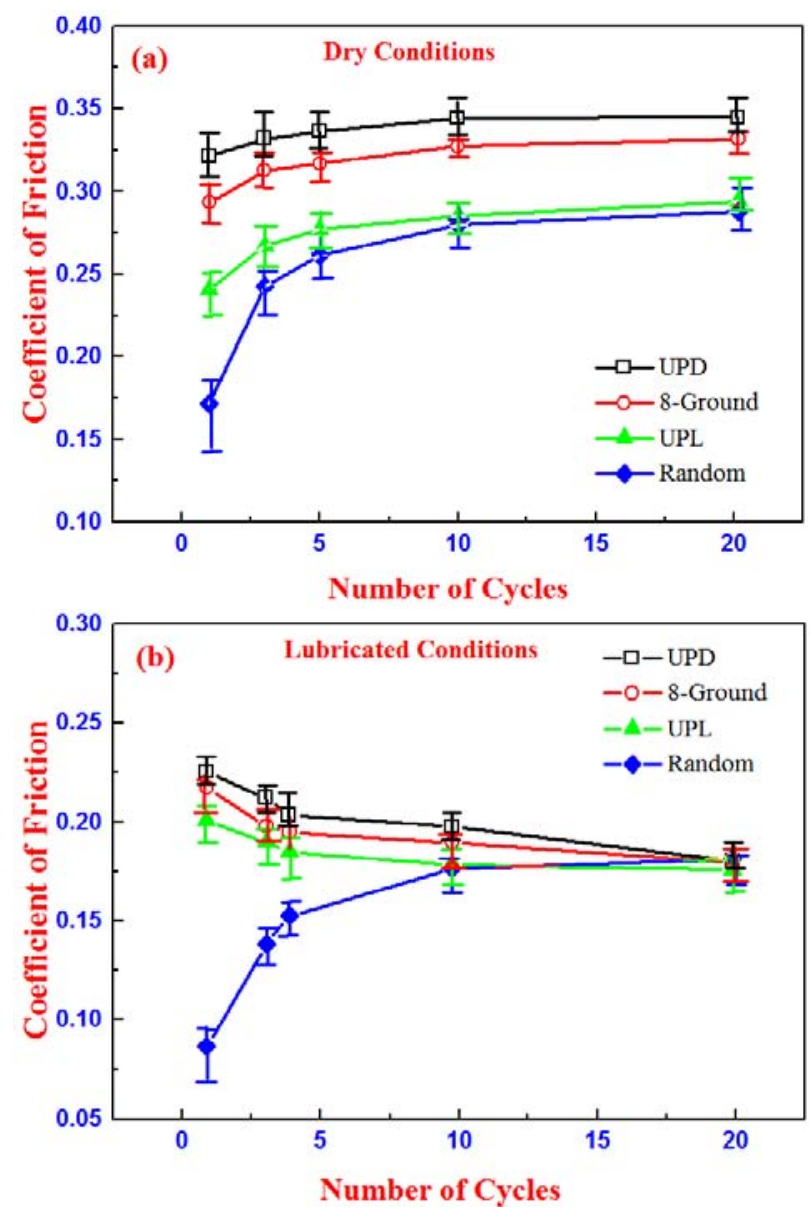

Fig. 1: Variation of average coefficient of friction with number of cycles for different surface textures under (a) dry and (b) lubricated conditions.

Previous analyses have shown [3] that the higher coefficient of friction values for the UPD surfaces was attributed to the 
constrained nature of flow for the softer pin material during sliding. For the random surface case, the coefficient of friction values were lower as the flow was unconstrained and provided less resistance to the pin during sliding. The constraint to flow offered by the 8-ground and UPL surfaces were expected to fall in between the UPD and the random surfaces [3].

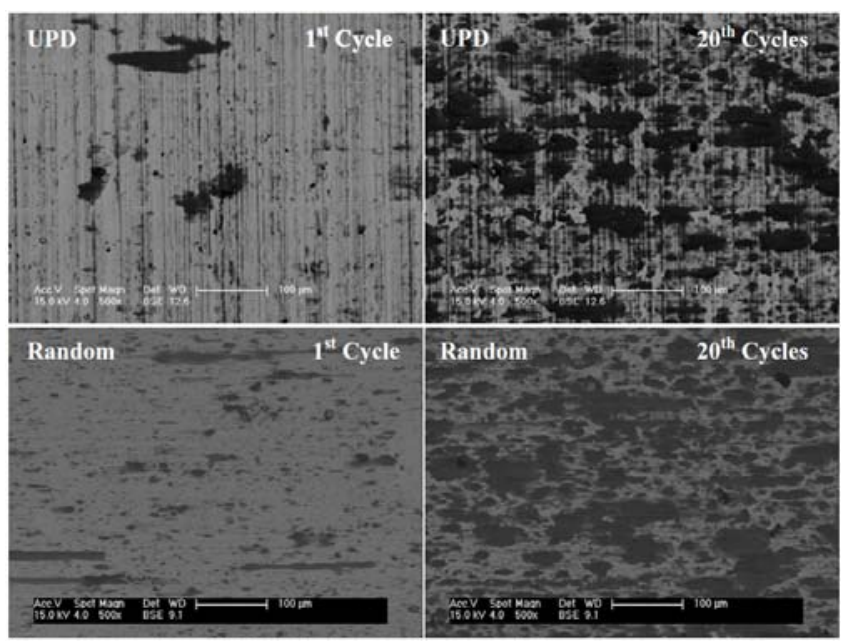

Fig. 2: SEM of UPD and random surfaces tested under dry conditions for $1^{\text {st }}$ and $20^{\text {th }}$ cycles.

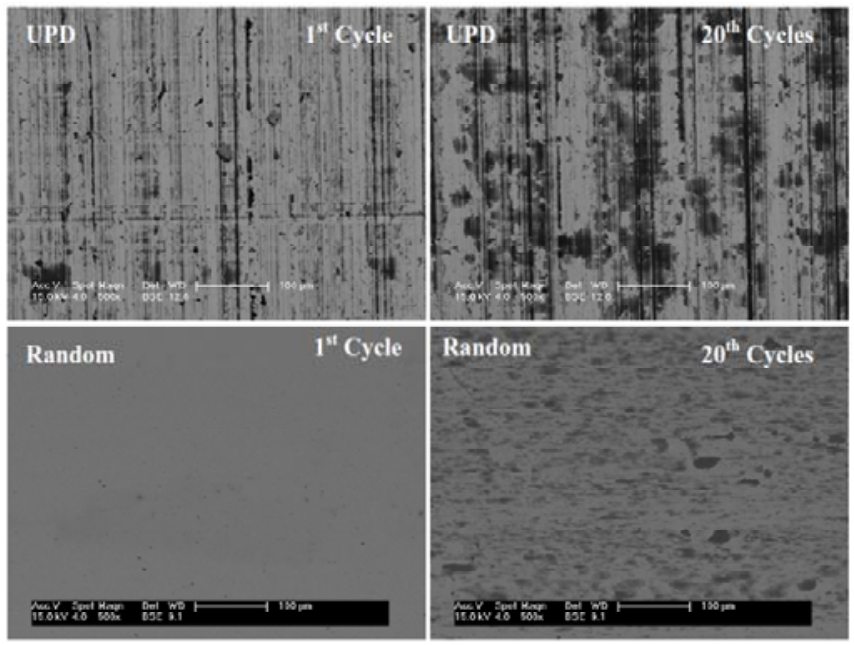

Fig. 3: SEM of UPD and random surfaces tested under lubricated conditions for $1^{\text {st }}$ and $20^{\text {th }}$ cycles.

In the present investigation, under dry conditions, the coefficient of friction increases with number of cycles. It was also observed that the transfer layer formation increases with increasing number of cycles. The transfer layer formation plays an important role in controlling the frictional phenomena. The presence of transfer layer on the steel plate during each cycles leads to sliding against similar pairs and thus the adhesion component [3] of friction increases with number of cycles. In addition, each sliding cycle work-hardens the contacting interface. The work-hardened transfer layer acts as third-body abrasive medium during sliding. Hence, both coefficient of friction and transfer layer increases with number of cycles. Under lubricated conditions, the amount of transfer layer formation is less and also the work-hardening is less. The addition of lubrication at the interface also minimizes the adhesion component of friction. Hence, the lower amount of transfer layer fills the asperities of the steel plate at lower number of cycles. At higher number of cycles they form a unique surface structure (comprises of transfer layer and unidirectional grinding marks along the sliding direction) due to self-organization and thus all surfaces shows a steady state friction values as shown in Fig. 1.

\section{CONCLUSIONS}

The conclusions based on experimental results are as follows.

- The coefficient of friction and transfer layer formation under both dry and lubricated conditions during the first few cycles depended on the die surface textures. Thereafter, friction became less dependent of the surface texture.

- $\quad$ The coefficient of friction increased with number of cycles under dry conditions for all kinds of textures. This was attributed to increased adhesion with cycle number and work-hardening of the transfer layer which acted as thirdbody abrasive medium.

- Under lubricated conditions, the adhesion component is less. In addition, the amount of transfer layer formation was less; hence, it fills the asperities of the steel plate at the lower number of cycles and forms a unique surface structure at the higher number of cycles.

- The variation in the coefficient of friction was attributed to the self-organization of surfaces during sliding.

\section{REFERENCES}

[1] Fox-Rabinovich, G. S., Totten, G. E., 2006 "Selforganization during Friction," CRC Press.

[2] Mortazavi, V., Menezes, P. L., and Nosonovsky, M., 2011, "Studies of Shannon Entropy Evolution due to Selforganization during the Running-in," ASME/STLE International Joint Tribology Conference (IJTC2011), Los Angeles, USA, pp. 303-305.

[3] Menezes, P. L., Kishore and Kailas, S. V., 2006, "Influence of Surface Texture on Coefficient of Friction and Transfer Layer Formation During Sliding of Pure Magnesium Pin on 080 M40 (EN8) Steel Plate,” Wear, 261, pp. 578-591.

[4] Menezes, P. L., Kishore, Kailas, S. V., and Lovell, M. R., 2011, "The Role of Surface Texture on Friction and Transfer Layer Formation during Repeated Sliding of Al4Mg against Steel,” Wear, 271, pp. 1785-1793. 